\title{
A Continuous-Flow Model for in vitro Cultivation of Mixed Microbial Populations Associated With Cystic Fibrosis Airway Infections
}

\author{
Thomas James O'Brien and Martin Welch* \\ Department of Biochemistry, University of Cambridge, Cambridge, United Kingdom
}

The airways of people with cystic fibrosis (CF) provide a nutrient-rich environment which favours colonisation by a variety of bacteria and fungi. Although the dominant pathogen associated with CF airway infections is Pseudomonas aeruginosa, it is becoming increasingly clear that inter-species interactions between $P$. aeruginosa and other colonists in the airways may have a large impact on microbial physiology and virulence. However, there are currently no suitable experimental models that permit longterm co-culture of $P$. aeruginosa with other CF-associated pathogens. Here, we redress this problem by describing a "3R's-compliant" continuous-flow in vitro culture model

OPEN ACCESS

Edited by: Giovanna Batoni, University of Pisa, Italy

Reviewed by: Giordano Rampioni,

Roma Tre University, Italy Emanuela Frangipani,

University of Urbino Carlo Bo, Italy

${ }^{*}$ Correspondence: Martin Welch mw240@cam.ac.uk

Specialty section:

This article was submitted to Microbial Physiology and Metabolism, a section of the journal

Frontiers in Microbiology

Received: 17 September 2019 Accepted: 08 November 2019

Published: 22 November 2019

Citation:

O'Brien TJ and Welch M (2019) A Continuous-Flow Model for in vitro Cultivation of Mixed Microbial Populations Associated With Cystic

Fibrosis Airway Infections.

Front. Microbiol. 10:2713.

doi: 10.3389/fmicb.2019.02713 which enables long-term co-culture of three representative CF-associated microbes: P. aeruginosa, Staphylococcus aureus and Candida albicans. Although these species rapidly out-compete one another when grown together or in pairs in batch culture, we show that in a continuously-fed setup, they can be maintained in a very stable, steady-state community. We use our system to show that even numerically $(0.1 \%)$ minor species can have a major impact on intercellular signalling by $P$. aeruginosa. Importantly, we also show that co-culturing does not appear to influence species mutation rates, further reinforcing the notion that the system favours stability rather than divergence. The model is experimentally tractable and offers an inexpensive yet robust means of investigating inter-species interactions between CF pathogens.

Keywords: cystic fibrosis, continuous-flow, co-culture, in vitro, Pseudomonas aeruginosa, Staphylococcus aureus, Candida albicans

\section{INTRODUCTION}

Cystic fibrosis (CF) is the most common life-limiting genetic disorder within the Caucasian population (Cystic Fibrosis Foundation, 2019), with 1 in 40 people estimated to carry the common $\triangle \mathrm{F} 508$ mutation in the $\mathrm{CF}$ transmembrane conductance regulator (CFTR) gene (Bobadilla et al., 2002; Cystic Fibrosis Mutation Database, 2019). The most striking consequence of dysfunctional CFTR activity is the overproduction of nutrient-rich, mucilaginous sputum. This blocks the airways and generates a heterogenous environment with steep oxygen gradients and a lowered pH (Boucher, 2002; Tate et al., 2002; Worlitzsch et al., 2002). This environmental niche is rich in nutrients such as mucin, amino acids, iron, and nitrate making the $\mathrm{CF}$ airway prone to colonisation by a variety of microbial species (Grasemann et al., 1998; Jones et al., 2000; Palmer et al., 2007; Ghio et al., 2013). 
The resulting infections often persist for decades, leading to respiratory failure and eventually, premature death (Lyczak et al., 2002; Rajan and Saiman, 2002; Carmody et al., 2013, 2015; Elborn, 2016). Traditionally, these CF-associated infections have been linked with a relatively small number of easily-culturable pathogens, such as Pseudomonas aeruginosa or Staphylococcus aureus. However, the introduction of culture-independent molecular profiling approaches revealed that expectorated CF sputum samples often contain a much wider range of bacterial and fungal species (Sibley et al., 2006, 2008; Rogers et al., 2010a; Zhao et al., 2012; Carmody et al., 2013, 2015; Short et al., 2014; Boutin et al., 2015). This suggests that the CF airways may harbour a highly-diverse microbial community, although this notion has been challenged recently through the direct sampling of lavage fluid from the lungs of CF children. These new data suggest that to a large extent, the diversity of the previously reported CF-associated microbiome arises from contamination of the sample during passage through the oral cavity, and following sample processing (Jorth et al., 2019). Nevertheless, these newer studies still suggest that the CF airways harbour a core population of "non-conventional" pathogens, including Prevotella, Veillonella and Staphylococcus species, as well as “traditional CF pathogens" such as P. aeruginosa (PA).

The polymicrobial character of many CF-associated airway infections makes it crucial to consider what impact inter-species interactions have on the physiology and composition of the microbial consortium. Previous reports demonstrate that coculturing bacterial species in vitro and in vivo causes significant alterations in gene essentiality (Ibberson et al., 2017). This can lead to changes in microbial lifestyle, impacting upon the expression of virulence factors (Rogers et al., 2009, 2010a,b; Hibbing et al., 2010; Leekha et al., 2011; Elias and Banin, 2012; Quinn et al., 2014; Limoli et al., 2016). For example, PA senses peptidoglycan shed from Gram-positive bacteria, and this stimulates the production of extracellular lytic virulence factors (Korgaonkar et al., 2013). Polymicrobial communities also display altered responses to therapeutic intervention. This may explain why many of the currently used clinical interventions designed to target PA show varying degrees of efficacy between patients (Lopes et al., 2012; Peters et al., 2012). As a consequence of these conceptual realisations, research focus is gradually moving away from studying individual species in isolation toward co-cultivating the major CF associated pathogens (Spasenovski et al., 2010; Bragonzi et al., 2012; Filkins et al., 2015; Magalhães et al., 2016; Lopes et al., 2017; Makovcova et al., 2017). However, these efforts are hampered by the paucity of adequate polymicrobial infection models. The development of a model which enables the stable and longterm recapitulation of CF polymicrobial communities is therefore highly-desirable.

Here we describe the development of a simple in vitro continuous-flow co-culture model which utilises artificial sputum medium (ASM). ASM is known to physiologically recapitulate the nutritional composition of CF airway secretions (Palmer et al., 2007; Kirchner et al., 2012; Turner et al., 2015). To the best of our knowledge, our co-culture model (Figure 1) is the first to permit the long-term, steady-state co-culture of three distinct microbial species: PA, Staphylococcus aureus (SA), and Candida albicans (CA). Through viable cell counting and optical density measurements, we demonstrate that the abundance of each member of this microbial population remains unchanged over the course of 4 days and that a total carrying capacity can be reached and maintained within the culture vessel. By contrast, and in line with previous reports, when these species are co-cultured under batch conditions, PA rapidly outcompetes the other species (Machan et al., 1992; Duan et al., 2003; Hogan et al., 2004; Mashburn et al., 2005; McAlester et al., 2008; Cugini et al., 2010; Holcombe et al., 2010; Morales et al., 2010; Park et al., 2012; Korgaonkar et al., 2013; Baldan et al., 2014; Fugère et al., 2014; Rüger et al., 2014; Barnabie and Whiteley, 2015; Filkins et al., 2015; Nguyen et al., 2015; Zago et al., 2015; Nguyen and OglesbySherrouse, 2016). Our in vitro model provides a defined and experimentally tractable system which can be used to dissect interspecies interactions and determine the long-term impact of co-cultivation on the physiology and gene expression profiles of CF-associated pathogens. Our in vitro model also provides a robust and cheaper alternative to existing in vivo infection models, making it compliant with the current trend toward the refinement, replacement and reduction (3Rs) of animal models in research.

The species chosen for inoculation into our co-culture model represent three distinct classes of microorganisms; a Gramnegative species and dominant CF-associated pathogen (PA), a Gram-positive species, often associated with CF airway infections (SA) and a dimorphic fungus (CA), also commonly found in CF airway secretions (Conrad et al., 2013). As such, our continuousflow model lays a solid groundwork for the development and optimisation of an in vitro co-culture model which can be directly inoculated with expectorated CF sputum. This will hopefully enable, in the longer-term, full recapitulation of the CF-associated polymicrobial community in defined laboratory conditions.

\section{MATERIALS AND METHODS}

\section{Microbial Strains and Culture Conditions}

The bacterial/fungal strains used in this study are shown in Table 1. All bacterial strains were routinely cultured in lysogeny broth (LB) (Formedium) with vigorous aeration at $37^{\circ} \mathrm{C}$ overnight. Where necessary, cultures were supplemented with $50 \mu \mathrm{g} \mathrm{mL} \mathrm{m}^{-1}$ carbenicillin (to maintain pSB1057 in the $N$-(3-Oxododecanoyl)-L-homoserine lactone OdDHL biosensor strain) or $10 \mu \mathrm{g} \mathrm{mL}^{-1}$ tetracycline (to maintain pSB536 in the $N$-butanoyl-L-homoserine lactone BHL biosensor strain).

Artificial sputum medium was used for every mono-, dual-, and triple-species cultivation. ASM was made using a modified version of the recipe published by Turner et al. (2015). Briefly, bovine maxillary mucin was replaced with $1.25 \mathrm{~g} \mathrm{~L}^{-1}$ porcine stomach mucin type-II (Sigma-Aldrich) and salmon sperm DNA was replaced with $1 \mathrm{~g} \mathrm{~L}^{-1}$ fish sperm DNA (SigmaAldrich) as described by Kirchner et al. (2012). A detailed protocol for preparing ASM can be found in the Supplementary Information (SI.1). 


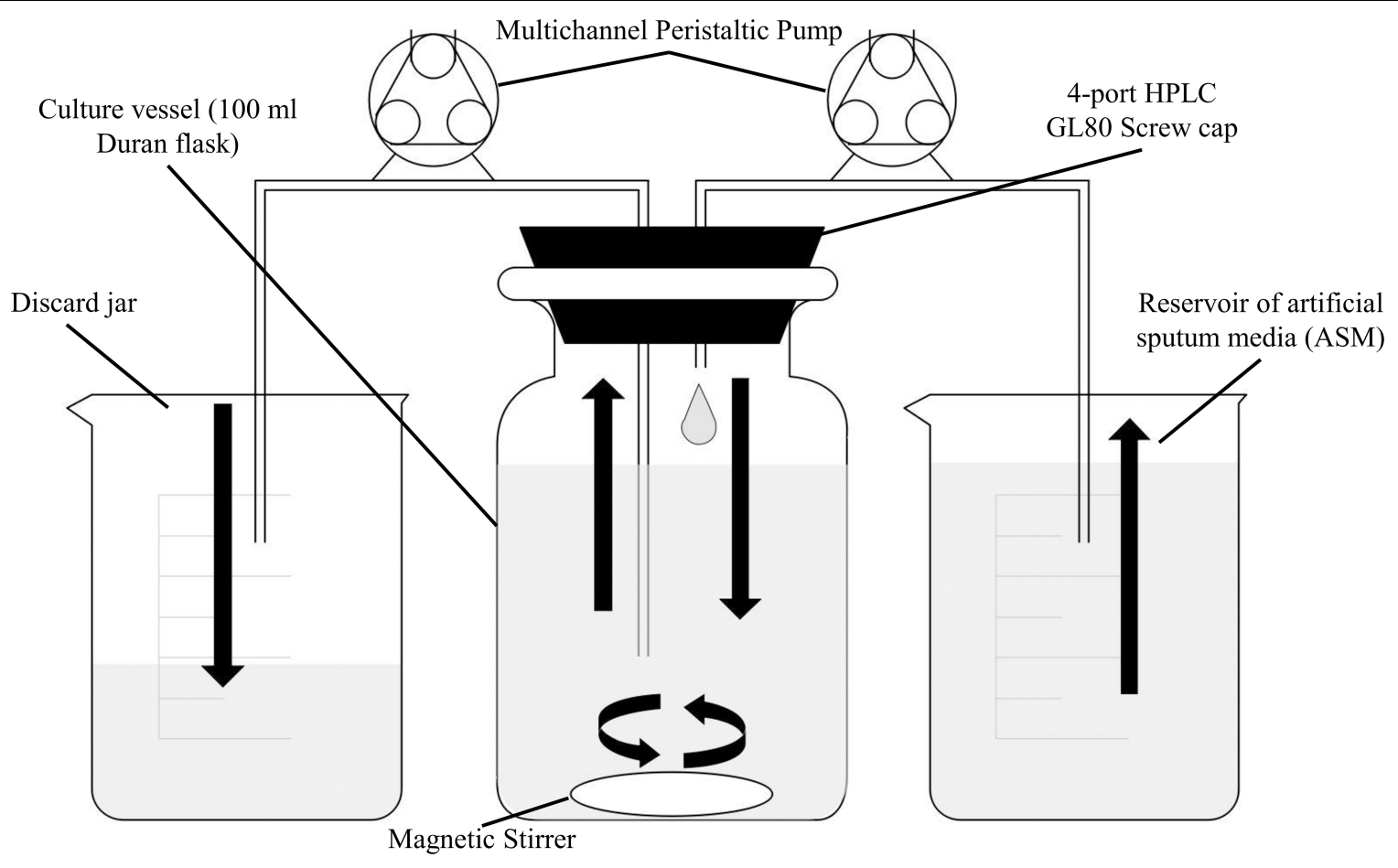

FIGURE 1 | Schematic diagram of the in vitro continuous-flow culture system used in this study. The main culture vessel (centre) is a $100 \mathrm{~mL}$ Duran bottle fitted with a 4-port HPLC GL80 screwcap lid, containing 4 sealable inlet/outlet ports. A multichannel peristaltic pump delivers fresh media (ASM) into the culture vessel from a reservoir, and also removes waste culture at the same rate of flow $(\mathrm{Q})$. Arrows show the direction of flow. The culture vessel is incubated at $37^{\circ} \mathrm{C}$ and the contents are kept homogenous through gentle stirring. If required, an in-line spectrophotometer fitted with a continuous-flow cuvette can be included in the setup prior to the discard jar. The value for $Q$ depends on the microbial species being cultured within the vessel.

\section{Continuous-Flow Culture Vessel and Culture Conditions}

A schematic of the continuous-flow culture system is shown in Figure 1. The culture vessel consists of a $100 \mathrm{~mL}$ flask (Duran), fitted with an assembled 4-port HPLC GL80 screw cap (Duran). A 24-channel IPC ISM934C standard-speed digital peristaltic pump (Ismatec) was used to deliver sterile ASM at a defined flow rate (Q) through $1.5 \mathrm{~mm}$ bore sterilin silicon tubing (Fisher Scientific) to the culture vessel. A different channel on the same pump was used to remove waste culture into a discard jar at the same flow-rate. The culture vessel was maintained at $37^{\circ} \mathrm{C}$ and its contents were kept homogenous by stirring (100 rpm) using a magnetic stir bar. When necessary, the culture optical density was monitored at $600 \mathrm{~nm}\left(\mathrm{OD}_{600 \mathrm{~nm}}\right)$ by passing the removed waste culture through an in-line $6715 \mathrm{UV}$ series spectrophotometer (Jenway) fitted with a continuous-flow cuvette.

Overnight cultures were washed three times in sterile $1 \times$ phosphate buffered saline (PBS, Oxoid) prior to inoculating the culture vessel. Pre-warmed ASM $(100 \mathrm{~mL})$ in the culture vessels was inoculated with the required combination of microbial species. Each species was introduced into the culture vessel to achieve a starting $\mathrm{OD}_{600 \mathrm{~nm}}$ of 0.05 . The vessel was incubated for $3 \mathrm{~h}$ prior to staring the flow of medium. For monospecies and co-culture experiments not containing CA, the flow rate (Q) was set at $170 \mu \mathrm{L} \mathrm{min}{ }^{-1}$. For co-culture experiments including $\mathrm{CA}, \mathrm{Q}$ was decreased to $145 \mu \mathrm{L} \min ^{-1}$. For all continuous-flow experiments, samples (1 mL volume) for cell enumeration were withdrawn using a syringe fitted with a sterile needle inserted through the rubber septa in the HPLC ports.

\section{Aerobic and Stirred Batch Culture Conditions}

For aerobic batch cultures, $250 \mathrm{~mL}$ Erlenmeyer flasks containing pre-warmed ASM (inoculated with the indicated strains to a starting $\mathrm{OD}_{600} \mathrm{~nm}$ of 0.05 ) were incubated at $37^{\circ} \mathrm{C}$ with vigorous shaking $(180 \mathrm{rpm})$. Stirred batch cultures were set up as described for the continuous-flow experiments (see section "ContinuousFlow Culture Vessel and Culture Conditions"), except with $\mathrm{Q}=0 \mu \mathrm{L} \mathrm{min}{ }^{-1}$. For both types of batch culture, samples $(1 \mathrm{~mL}$ volume) were taken from the culture vessel for $\mathrm{OD}_{600 \mathrm{~nm}}$ analysis and viable cell counting.

\section{Microbial CFU $\mathrm{mL}^{-1}$ Enumeration}

Colony forming units (CFU) per $\mathrm{mL}$ of culture were determined using the single plate-serial dilution spotting (SP-SDS), as described previously (Thomas et al., 2015). Serial dilutions were made in sterile PBS and $20 \mu \mathrm{L}$ of each dilution was spotted onto the appropriate selective agar. PA was isolated using pseudomonas agar base (Oxoid) supplemented with cetrimide $\left(200 \mu \mathrm{g} \mathrm{mL}^{-1}\right)$ and sodium nalidixate $\left(15 \mu \mathrm{g} \mathrm{mL}^{-1}\right)$. SA was isolated on mannitol salt agar (Oxoid). CA was isolated on BiGGY agar (Oxoid). During co-culture experiments involving $\mathrm{CA}$, the agar plates used to isolate PA and SA were further 
TABLE 1 | Microbial strains used in this study.

\begin{tabular}{|c|c|c|}
\hline Strain & Description & References \\
\hline PAO1 & $\begin{array}{l}\text { Pseudomonas aeruginosa, spontaneous chloramphenicol-resistant derivative. Used worldwide as a } \\
\text { laboratory reference strain (isolated Melbourne, 1954). }\end{array}$ & Holloway, 1955 \\
\hline ATCC 25923 & $\begin{array}{l}\text { Staphylococcus aureus Rosenbach (ATCC }{ }^{\circledR} 25923 \mathrm{D}-5^{\mathrm{TM}} \text { ), methicillin sensitive clinical isolate. Laboratory } \\
\text { reference strain lacking recombinases and } m e c A \text { (isolated Seattle, 1945). }\end{array}$ & Treangen et al., 2014 \\
\hline SC5314 & $\begin{array}{l}\text { Candida albicans, clinical isolate commonly used as a wild-type laboratory reference strain (isolated } \\
\text { New York, 1980s). }\end{array}$ & Gillum et al., 1984 \\
\hline PAO1 $\Delta p q s A$ CTX-lux::pqsA & $\begin{array}{l}\text { PQS biosensor strain. } \triangle p q s A \text { mutant of PAO1 containing a pqsA promoter:: luxCDABE fusion integrated at } \\
\text { a neutral site in the chromosome. }\end{array}$ & Fletcher et al., 2007 \\
\hline JM109 (pSB1057) & OdDHL biosensor strain. Escherichia coli JM109 containing pSB1057. & Winson et al., 1998 \\
\hline JM109 (pSB536) & BHL biosensor strain. Escherichia coli JM109 containing pSB536. & Winson et al., 1998 \\
\hline
\end{tabular}

supplemented with $5 \mu \mathrm{g} \mathrm{mL}^{-1}$ itraconazole to inhibit the growth of CA. All plates were incubated at $37^{\circ} \mathrm{C}$. Pseudomonas agar base and mannitol salt plates were incubated overnight $(16 \mathrm{~h})$. BiGGY agar plates were incubated for $24 \mathrm{~h}$. CFU mL ${ }^{-1}$ counts are averages taken from three technical repeats. There was no significant difference between total CFU $\mathrm{mL}^{-1}$ counts of pure microbial cultures plated onto either non-selective (LB-agar) or any of the selective agar (data not shown).

\section{Quantification of Quorum Sensing Molecules}

Aliquots $(1.5 \mathrm{~mL})$ of culture were collected after 24 and $96 \mathrm{~h}$ (as indicated) of incubation. The cells were pelleted by centrifugation $\left(15,000 \times g, 5 \mathrm{~min}, 20^{\circ} \mathrm{C}\right)$ and the supernatant was filtered (0.22 $\mu \mathrm{m}$ pore size). Aliquots of the supernatant were snap frozen in liquid $\mathrm{N}_{2}$ and stored at $-20^{\circ} \mathrm{C}$ until use. OdDHL was detected using JM109 (pSB1057). BHL was detected using JM109 (pSB536). PQS was detected using PAO1 $\Delta p q s A$ CTXlux::pqsA. Overnight starter cultures of the reporter strains were sub-cultured in LB supplemented with the appropriate antibiotics and grown to $\mathrm{OD}_{600 \mathrm{~nm}}=1.0$. Following this, aliquots $(60 \mu \mathrm{L}$ volume) of the normalised cell culture were transferred to a sterile clear-bottomed black opaque 96-well plate (Greiner BioOne) containing an equal volume of thawed culture supernatant. The plates were incubated at $30^{\circ} \mathrm{C}$ with shaking $(100 \mathrm{rpm})$ for $3 \mathrm{~h}$. Bioluminescence was recorded using a FLOUstar Omega plate reader (BMG). Standard curves to calibrate the biosensor outputs were constructed using known concentrations of synthetic quorum sensing molecules dissolved in ASM.

\section{Quantification of Pyocyanin}

Pyocyanin quantification was performed following chloroform extraction of the pigment (Knight et al., 1979). Aliquots $(10 \mathrm{~mL}$ volume) of culture were collected after $96 \mathrm{~h}$ growth and the cells were pelleted $\left(4000 \times \mathrm{g}, 30 \mathrm{~min}, 4^{\circ} \mathrm{C}\right)$. The culture supernatants were filter sterilised $(0.22 \mu \mathrm{m}$ pore size). Chloroform $(4.5 \mathrm{~mL})$ was added to $7.5 \mathrm{~mL}$ of the cell-free culture supernatant and the suspension was vigorously vortexed for $30 \mathrm{~s}$. The immiscible layers were separated by centrifugation $(4000 \times g, 10 \mathrm{~min}$, $4^{\circ} \mathrm{C}$ ). An aliquot ( $3 \mathrm{~mL}$ volume) of the blue-green chloroform phase was removed and mixed with $1.5 \mathrm{~mL} 0.2 \mathrm{M} \mathrm{HCl}$. The immiscible layers were then separated by centrifugation and
$1 \mathrm{~mL}$ of the rose-pink phase was transferred to a cuvette. The pyocyanin absorbance was measured at $520 \mathrm{~nm}$ using a BioSpectrometer Kinetic spectrophotometer (Eppendorf) and converted to concentration $\left(\mu \mathrm{g} \mathrm{mL}^{-1}\right)$ by multiplying the $\mathrm{A}_{520 \mathrm{~nm}}$ value by 26.6 .

\section{Estimation of Mutation Rates}

Mutation rates in the chemostat were measured as described by Foster (2006). Initially, the rate constant $(\lambda)$ associated with exponential growth of PA and SA co-cultured in ASM, was determined by enumeration of $\mathrm{CFU} \mathrm{mL}^{-1}$ on selective agar plates. Next, the total cell count $(N)$ within the culture vessel at steady-state growth was determined. The number of spontaneous rifampicin resistant PA or SA mutants, $r$, was measured after $\mathrm{t}_{1}=0 \mathrm{~h}, \mathrm{t}_{2}=24 \mathrm{~h}$ and $\mathrm{t}_{3}=96 \mathrm{~h}$ of incubation. Total cell numbers in the chemostat did not change appreciably between 24 and $96 \mathrm{~h}$. The value of $r$ was determined by plating aliquots (100 $\mu \mathrm{L}$ volume) of culture onto either pseudomonas isolation agar supplemented with $60 \mu \mathrm{g} \mathrm{mL}^{-1}$ rifampicin (for PA), or mannitol salt agar supplemented with $0.05 \mu \mathrm{g} \mathrm{mL}^{-1}$ rifampicin (for SA). The mutation rate per cell per generation $(\mathrm{m})$ was calculated according to Eq. 2 in Foster (2006);

$$
\mu=\frac{1}{N \lambda} \frac{\left(r_{2}-r_{1}\right)}{\left(t_{2}-t_{1}\right)}
$$

\section{Statistical Analysis}

Unless otherwise stated, all data represent the mean $\pm \mathrm{SD}$ of three independent biological experiments. Results were analysed by one-way or two-way ANOVA (as indicated), or Student's unpaired $t$-test using GraphPad Prism version 8.2.0, with $P<0.05$ being considered statistically significant.

\section{RESULTS}

\section{Mono-Species Continuous-Flow Culture (PA01)}

As a first step, we confirmed that PA, SA and CA could all grow in ASM. This was done by inoculating each species into flat-bottomed microtitre plates containing ASM. The plates were incubated at $37^{\circ} \mathrm{C}$ with vigorous shaking $(180 \mathrm{rpm})$ in a FluoStar Omega plate reader, and the culture optical density $\left(\mathrm{OD}_{600}\right)$ 

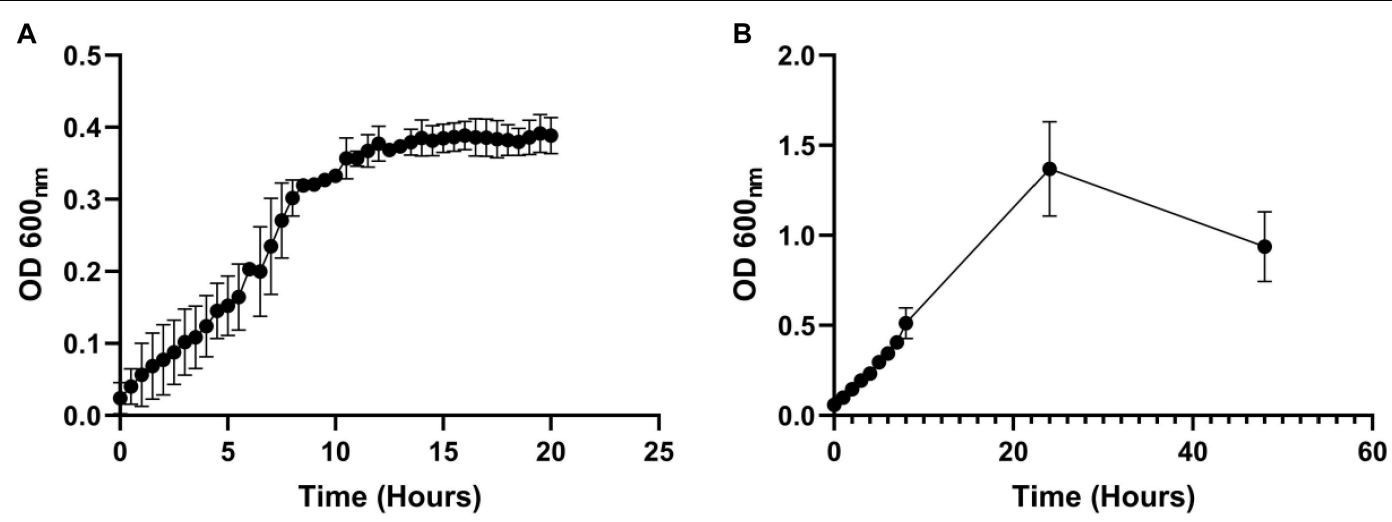

FIGURE 2 | Growth of $P$. aeruginosa in ASM in batch and continuous flow culture conditions. Growth (monitored as $\mathrm{OD}_{600}$ ) of PA in ASM during (A) continuous-flow culture $\left(Q=170 \mu \mathrm{L} \mathrm{min}{ }^{-1}\right)$; (B) batch culture $\left(Q=0 \mu \mathrm{L} \mathrm{min}{ }^{-1}\right)$. Data represent the mean \pm standard deviation from three independent experiments.

was monitored every $15 \mathrm{~min}$. PA, SA and CA grew rapidly in ASM, achieving a final $\mathrm{OD}_{600}$ of $>1$ after $24 \mathrm{~h}$ in all cases (Supplementary Figure S1).

Next, we measured whether a mono-species culture of PA could be maintained with stable steady-state titres in our continuous-flow setup. The laboratory reference strain, PAO1, was inoculated into the continuous-flow system using a flow-rate $\mathrm{Q}=170 \mu \mathrm{L} \mathrm{min}{ }^{-1}$ and the $\mathrm{OD}_{600}$ was measured every $30 \mathrm{~min}$ as described in Section "Continuous-Flow Culture Vessel and Culture Conditions." The $\mathrm{OD}_{600}$ increased almost linearly for the first $8 \mathrm{~h}$ and then reached a plateau $\left(\mathrm{OD}_{600} \approx 0.4\right)$ after $10 \mathrm{~h}$ of incubation (Figure 2A). By comparison, during growth in the same medium and experimental setup with $\mathrm{Q}=0 \mu \mathrm{L} \mathrm{min}^{-1}$ (i.e., in stirred batch mode), the PA culture reached a final $\mathrm{OD}_{600}$ of $>1$ (Figure 2B). We conclude that during continuous-flow operation, the setup allows the culture to achieve a steady-state carrying capacity with an $\mathrm{OD}_{600}$ well-below the final $\mathrm{OD}_{600}$ associated with entry into the stationary phase of growth in the same medium.

\section{Dual-Species Co-culture (PA-SA)}

Staphylococcus aureus is also associated with CF airway infection and is particularly prevalent in adolescent patients (Goss and Muhlebach, 2011; Conrad et al., 2013; Jorth et al., 2019). Despite PA and SA being frequently co-isolated from CF patients, numerous antagonistic interactions have been identified between these species, and PA readily outcompetes SA in vitro in mixed cultures (Machan et al., 1992; Duan et al., 2003; Mashburn et al., 2005; Park et al., 2012; Korgaonkar et al., 2013; Baldan et al., 2014; Fugère et al., 2014; Rüger et al., 2014; Filkins et al., 2015; Nguyen et al., 2015). We therefore wanted to determine if the two species could be stably maintained in our continuous-flow culture system. We found that a mixed species co-culture of PA and SA could be readily maintained to yield an apparently stable steady-state composition using $\mathrm{Q}=170 \mu \mathrm{L} \mathrm{min}-1$. The $\mathrm{CFU}$ counts for each species are shown in Figure $\mathbf{3 A}$, and the co-culture $\mathrm{OD}_{600}$ measurements are shown in Supplementary Figure S2. A steady state composition of around $10^{7} \mathrm{SA}$ CFU $\mathrm{mL}^{-1}$ and $10^{8} \mathrm{PACFU} \mathrm{\textrm {mL } ^ { - 1 }}$ was established by $24 \mathrm{~h}$ of growth, and there were no significant differences in the viable cell counts following this $(P>0.05)$ up to $96 \mathrm{~h}$ of growth. By contrast, during aerobic batch culture in flasks, PA rapidly outcompeted SA and no viable SA could be recovered at the $96 \mathrm{~h}$ sampling point (Figure 3B). PA also outcompeted SA during stirred batch

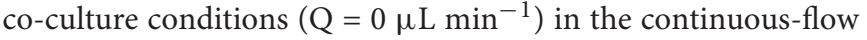
vessel (Figure 3C), albeit at a slower rate. Taken together, these data indicate that a continual supply of fresh media and removal of waste products is crucial for permitting a successful PA-SA co-culture in vitro.

\section{Dual-Species Co-culture (PA-CA)}

Fungi, such as Candida sp. and Aspergillus sp. are also associated with CF airway infections (Williams et al., 2016; Bouchara et al., 2018). We therefore examined whether Candida albicans (CA) could be maintained alongside PA in the continuous-flow setup. This is important because inter-kingdom interactions between PA and CA have been previously shown to affect virulence factor production by both species (Hogan et al., 2004; McAlester et al., 2008; Cugini et al., 2010; Holcombe et al., 2010). We found that a co-culture of PA and CA could be readily maintained in the continuous-flow setup (Figure 4A), although to prevent a washout of CA from the culture vessel over time we had to decrease

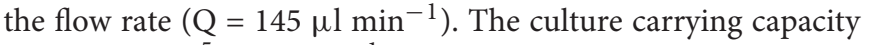

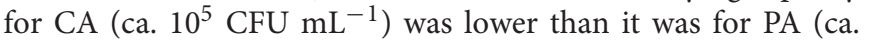
$\left.10^{8} \mathrm{CFU} \mathrm{mL} \mathrm{mL}^{-1}\right)$, but once a steady-state had been achieved (after $24 \mathrm{~h}$ incubation) no statistically significant differences in PA or CA viable cell counts were observed $(P>0.05)$. In contrast, CA titres rapidly declined during aerobic batch coculture (Figure 4B). A similar, albeit slower decline in CA titres was observed during stirred batch growth (Figure 4C).

\section{Dual-Species Co-culture (SA-CA)}

We next wanted to confirm that a stable co-culture of SA and CA could be maintained independent of PA. Using $\mathrm{Q}=145 \mu \mathrm{L} \mathrm{min}-1$, this was indeed the case (Figure 5A), and after $24 \mathrm{~h}$ growth, the ratio of SA:CA remained essentially unchanged. As in the PA-CA co-culture, at steady-state, the carrying capacity (ca. $10^{5} \mathrm{CFU} \mathrm{mL}^{-1}$ ) for CA was lower than 

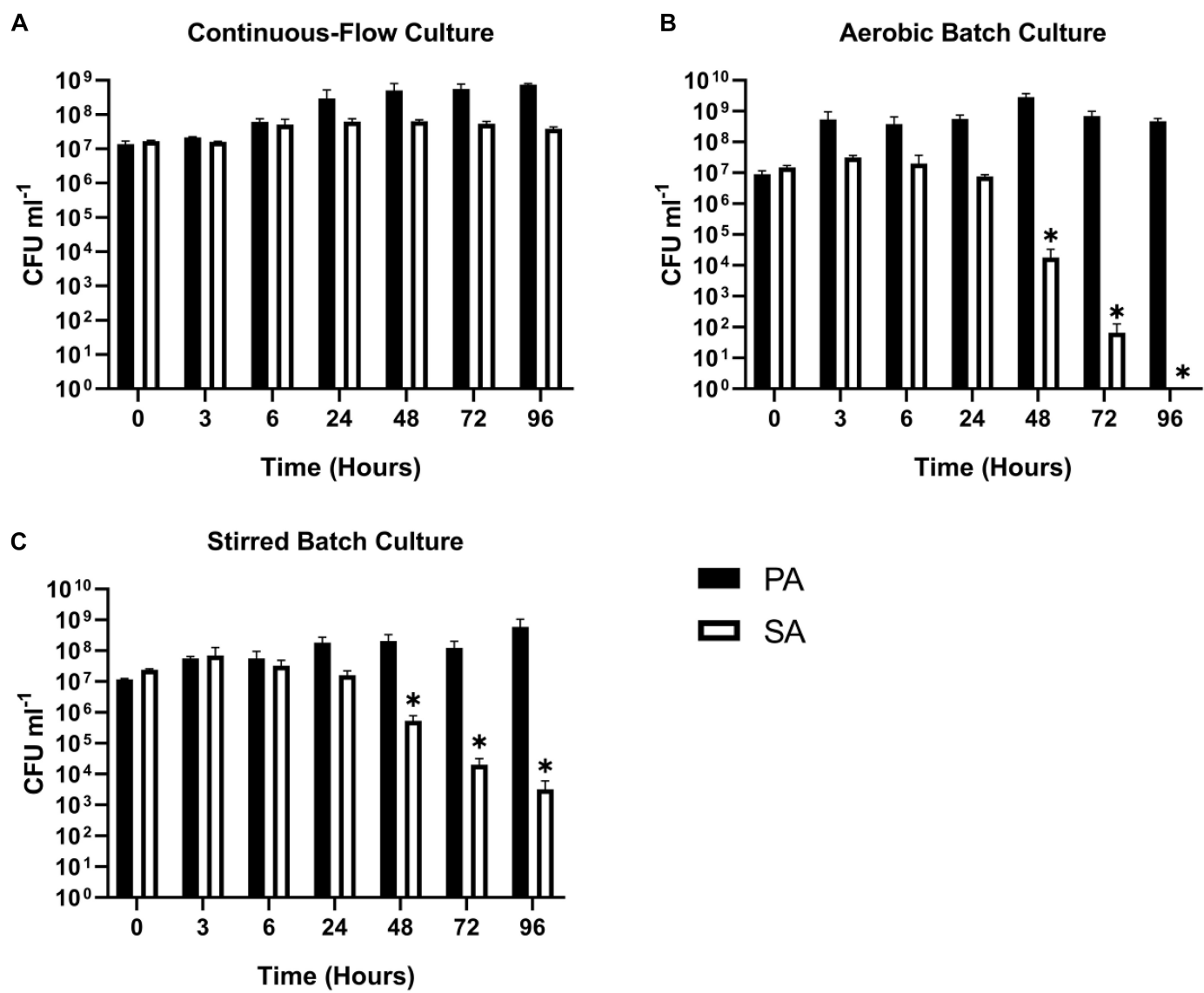

FIGURE 3 | Continuous-flow culture allows P. aeruginosa and S. aureus to be maintained in a stable steady-state. Viable cell counts [colony forming units (CFUs)] of P. aeruginosa PAO1 (PA, black bars) and S. aureus 25923 (SA, white bars) during co-culture in ASM using: (A) a continuous-flow setup; (B) aerobic batch culture; and $\mathbf{( C )}$ stirred batch culture. Data represent as mean \pm standard deviation from three independent experiments. Asterisks represent significant $\left({ }^{*} P<0.05\right)$ differences in CFU $\mathrm{mL}^{-1}$ counts in comparison with the data from the $24 \mathrm{~h}$ time point.

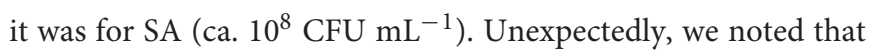
following aerobic and stirred batch culture, the CA outcompeted the SA (Figures 5B,C). This confirms that in mixed cultures, a species comprising just $0.1 \%$ of the microbiota can potentially have a major impact on titres of the [initially] numericallydominant organism.

\section{Triple-Species Co-culture}

With the continuous-flow culture system clearly capable of maintaining dual-species co-cultures of PA-SA, PA-CA and CA$\mathrm{SA}$, we next wanted to determine if all three species could be co-cultured to achieve a stable steady-state composition. We found that setting $\mathrm{Q}=145 \mu \mathrm{L} \mathrm{min}^{-1}$, a mixed population of all three microbial species could be maintained at a steady state for $96 \mathrm{~h}$ of incubation (Figure 6A; the corresponding in-line $\mathrm{OD}_{600}$ data are shown in Supplementary Figure S3). Once the steadystate had been achieved (i.e., after $24 \mathrm{~h}$ of growth) there were no significant differences in the CFU mL $\mathrm{mL}^{-1}$ counts for each species for the remaining duration of the co-culture $(P>0.1)$. The $\mathrm{PA}$ and SA titres remained at around $10^{8}-10^{9} \mathrm{CFU} \mathrm{mL} \mathrm{m}^{-1}$, and the CA titres remained at around $10^{5} \mathrm{CFU} \mathrm{mL}^{-1}$. By contrast, when co-cultured in aerobic batch culture, both SA and CA were outcompeted by PA (Figure 6B). Indeed, there was a progressive decrease in the number of SA CFUs in each of the samples harvested after the $24 \mathrm{~h}$ time-point $(P<0.0005)$, and by $72 \mathrm{~h}$, no viable $\mathrm{CA} C F U$ could be recovered. However, and unlike the PA-SA aerobic dual cultures (Figure 3B), SA could still be recovered at the $96 \mathrm{~h}$ sampling point, suggesting that the presence of CA affords a degree of protection, perhaps by decreasing the direct competition between PA and SA for shared resources. The stirred batch co-cultures yielded a somewhat different pattern (Figure 6C). Here, following the $24 \mathrm{~h}$ sampling point, PA titres remained high (ca. $10^{9}-10^{10} \mathrm{CFU} \mathrm{\textrm {mL } ^ { - 1 }}$ ) and constant, but there was a significant and progressive decrease in SA titres $(P<0.05)$. Unlike the aerobic batch culture, this was accompanied by a much slower decline in CA titres.

\section{Quantification of $P$. aeruginosa Quorum Sensing Molecules}

Quorum sensing (QS) mediated signalling pathways are linked to the regulation of secondary metabolite and extracellular virulence factor production by PA. Some of these QS-regulated 
A

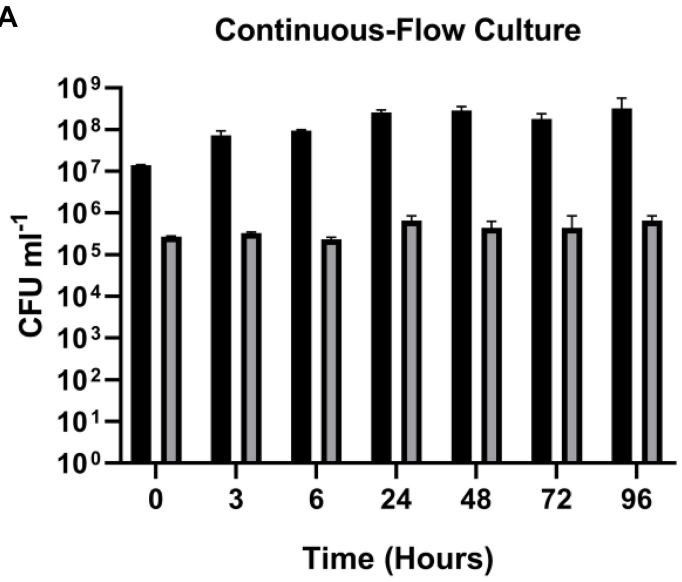

C

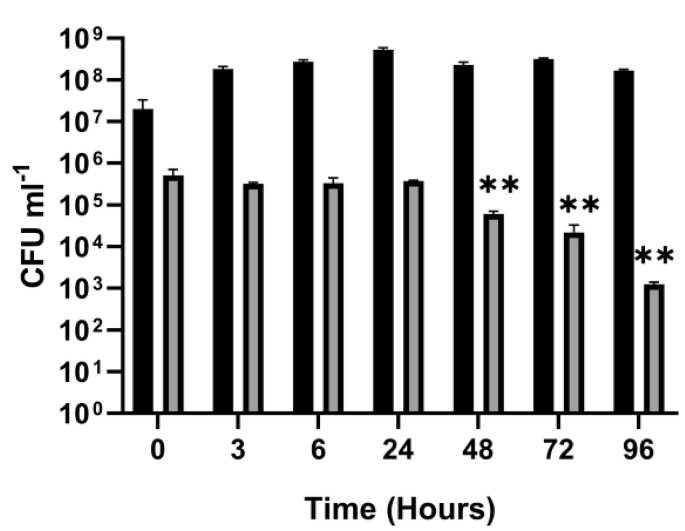

B

B Aerobic Batch Culture

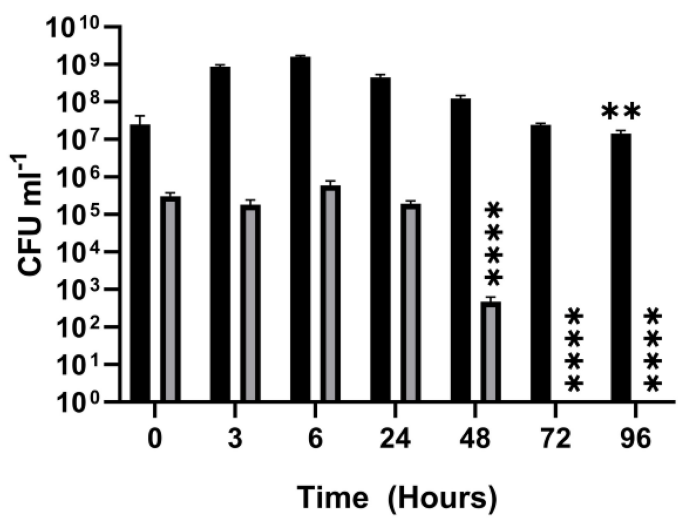

PA

FIGURE 4 | Continuous-flow culture allows $P$. aeruginosa and C. albicans to be maintained in a stable steady-state. Viable cell counts (CFU) of $P$. aeruginosa PAO1 (PA, black bars) and C. albicans SC5314 (CA, grey bars) during co-culture in ASM using: (A) a continuous-flow setup; (B) aerobic batch culture; and (C) stirred batch culture. Data represent as mean \pm standard deviation from three independent experiments. Asterisks represent significant differences in $\mathrm{CFU} \mathrm{mL}^{-1} \mathrm{counts}$ in comparison with the data from the $24 \mathrm{~h}$ time point $\left(* * P<0.005,{ }^{* * * *} P<0.0001\right)$.

factors have been implicated in mediating interactions with other microbial species (Gambello et al., 1993; Smith and Iglewski, 2003; Lau et al., 2004; Schuster and Greenberg, 2006; Dekimpe and Déziel, 2009; Antunes et al., 2010). To examine how other microbial species might impinge on QS in PA, we therefore determined the concentration of the Pseudomonas quinolone signal (PQS), N-(3-Oxododecanoyl)-L-homoserine lactone (OdDHL) and $N$-butanoyl-L-homoserine lactone (BHL) in the culture supernatant of single and mixed species co-cultures (Figures 7A-C, respectively).

The concentration of all three QS molecules was significantly $(P<0.0001)$ lower in the continuous-flow setup compared with the aerobic- and stirred-batch cultures. In the continuous-flow setup, there was no significant difference in the concentration of PQS between the 24 and $96 \mathrm{~h}$ sampling points, or of OdDHL between these sampling points $(P>0.1)$, although we did note an increase in BHL concentration in the PA-CA co-culture over this period $(P>0.05)$. In contrast, QS molecules accrued to much higher concentrations in the aerobic- and stirred-batch cultures. Moreover, the presence of co-cultivated species had a large, but differential impact on QS molecule production by PA. For example, in batch culture, SA appeared to stimulate OdDHL production, whereas CA appeared to depress OdDHL levels and stimulate PQS (and to a lesser extent, also BHL) production. Taken together, our data indicate that QS molecules accumulate to a much lower concentration in the continuous-flow setup compared with batch cultures.

\section{Quantification of Pyocyanin}

Pyocyanin is a redox-active PA secondary metabolite, and is linked with virulence and competition between microbial species in the CF lung (Castric, 1975; Hoffman et al., 2006; Voggu et al., 2006; Biswas et al., 2009; Filkins et al., 2015; Noto et al., 2017). We measured pyocyanin levels in the different culture setups at the endpoint of each experiment (Figure 8). Pyocyanin concentrations were significantly lower for all microbial species combinations in the continuous-flow setup compared with the aerobic- or stirred-batch cultures $(P<0.0001)$. No significant differences were observed in pyocyanin accumulation between the different microbial co-culture combinations following growth 


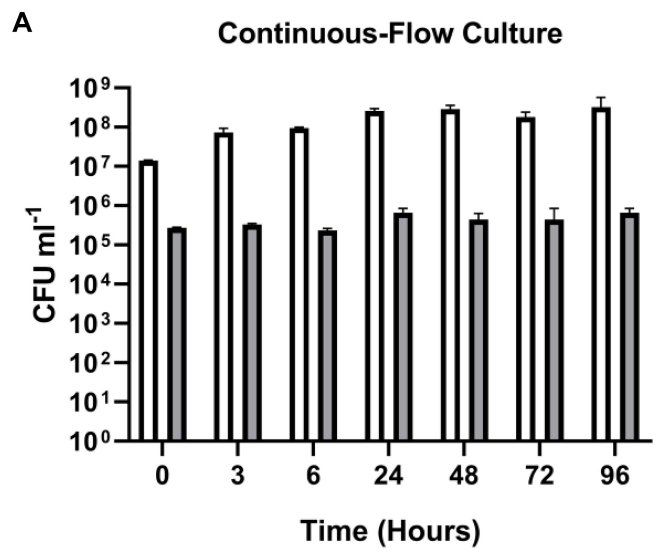

C

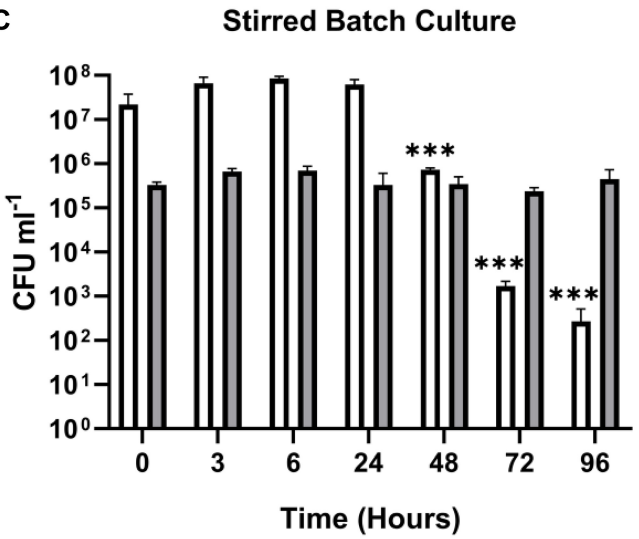

B

Aerobic Batch Culture

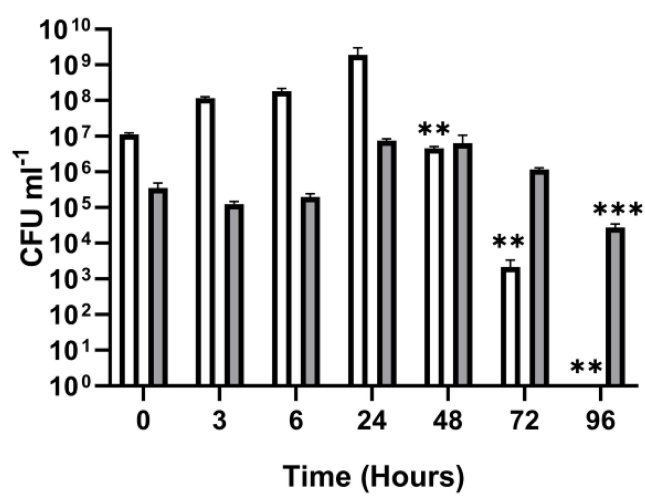

$\square S A$

ㄷA

FIGURE 5 | Continuous-flow culture allows S. aureus and C. albicans to be maintained in a stable steady-state. Viable cell counts of S. aureus 25923 (SA, white bars) and C. albicans SC5314 (CA, grey bars) co-cultures in ASM in: (A) a continuous-flow setup; (B) aerobic batch culture; and (C) stirred batch culture. Data represented as mean \pm standard deviation of three independent experiments. CFU mL ${ }^{-1}$ values are plotted on a $\log _{10}$ scale and asterisks represent significant differences in CFU mL ${ }^{-1}$ counts in comparison with the data from the $24 \mathrm{~h}$ time point $\left({ }^{*} P<0.005,{ }^{* * *} P<0.001,{ }^{* * * *} P<0.0001\right)$.

in the continuous-flow setup $(P>0.3)$. However, we did note that in the batch cultures, the presence of CA depressed pyocyanin accumulation.

\section{Estimation of Mutation Rates in Co-cultures of $P$. aeruginosa and}

\section{S. aureus}

One possible use of the continuous-flow system described here would be to investigate how the presence of co-habiting species affects evolutionary trajectory(s). To gauge this, we measured the mutation rate of each species during co-culture. Mutation rates were measured as described by Foster (2006) and were assessed shortly after the steady-state had been attained (i.e., at the $24 \mathrm{~h}$ time-point) and at the end of the experiment ( $96 \mathrm{~h}$ time-point). The mean number of $\mathrm{Rif}^{\mathrm{R}}$-conferring mutations per cell division was comparable with previously-reported values $\left[\approx 10^{-8}-10^{-9}\right.$ mutations/cell/division (Schaaff et al., 2002; Dettman et al., 2016)] and was consistently low for both PA and SA, with no statistically significant differences between the 24 and $96 \mathrm{~h}$ sampling points $(P>0.1)$ (Figure 9). We conclude that PA and SA do not exhibit abnormal mutability in the continuous-flow setup and that co-culture of these species has no apparent impact on their respective mutation rate.

\section{Continuous-Flow Cultures Maintain a Constant pH}

We also examined the endpoint $\mathrm{pH}$ of mixed-species cultures to see whether this differed from the starting $\mathrm{pH}$ of ASM ( $\mathrm{pH}$ 6.7). We found that irrespective of the microbes and combinations of microbes being tested, the continuous flow cultures maintained a remarkably constant $\mathrm{pH}$ that was close to the starting $\mathrm{pH}$. Stirred batch cultures maintained a $\mathrm{pH}$ of $\mathrm{ca}$. 7 , whereas aerobic batch cultures exhibited an endpoint $\mathrm{pH}>8$ (Supplementary Figure S4).

\section{DISCUSSION}

In this work, we have shown that a simple in vitro continuousflow co-culture system enables long-term co-culture of three distinct microbial species (PA, SA and CA) associated with CF airway infections. When co-cultured in batch, these organisms ordinarily outcompete one another, leading to domination by 

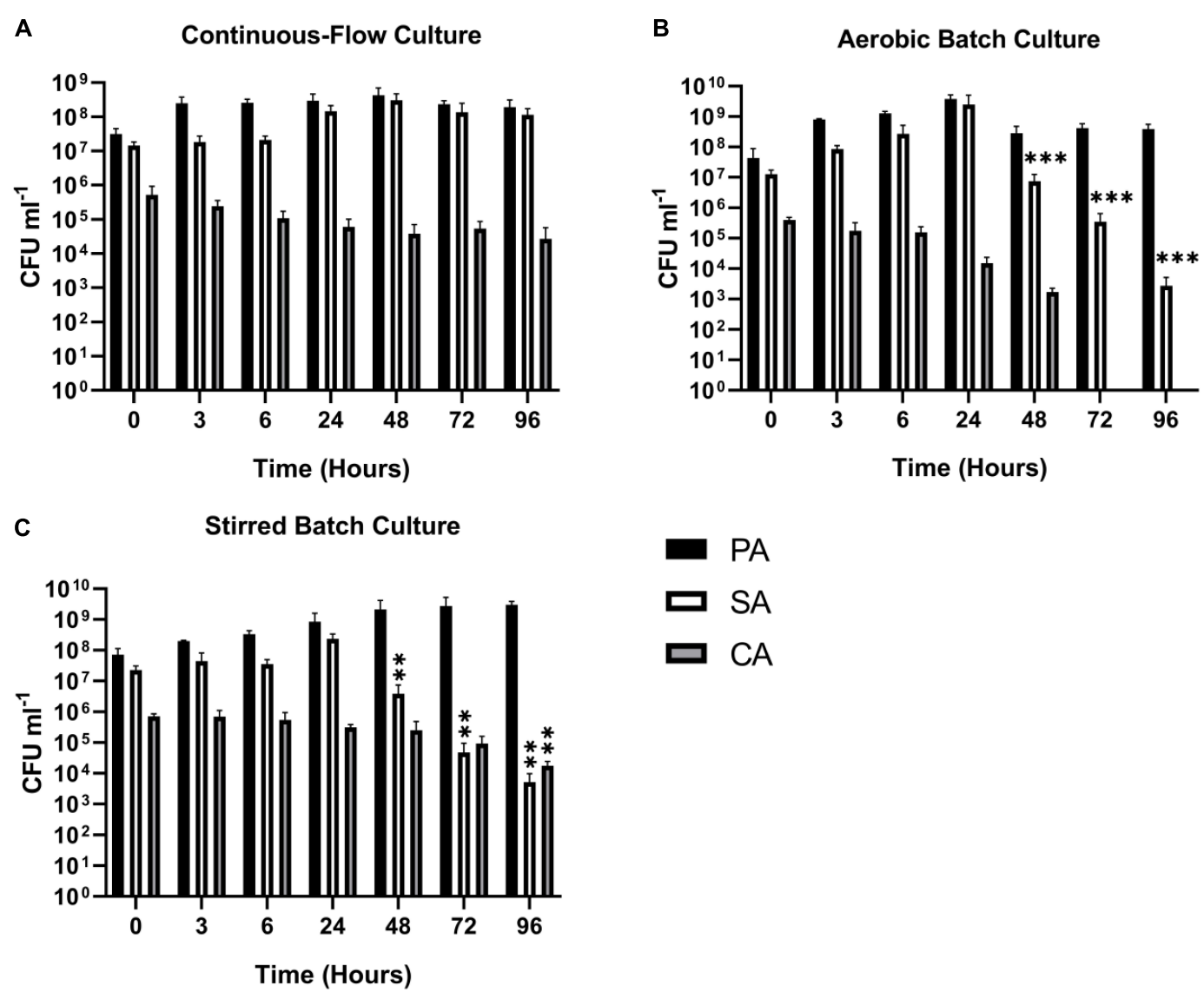

FIGURE 6 | Continuous-flow culture allows $P$. aeruginosa, C. albicans, and $S$. aureus to be maintained in a stable steady-state. Viable cell counts of $P$. aeruginosa PAO1 (PA, black bars) S. aureus 25923 (SA, white bars) and C. albicans SC5314 (CA, grey bars) co-cultures in ASM in: (A) a continuous-flow setup; (B) aerobic batch culture; and (C) stirred batch culture. Data represented as mean \pm standard deviation of three independent experiments. CFU mL ${ }^{-1}$ values are plotted on a $\log _{10}$ scale and asterisks represent significant differences in CFU mL ${ }^{-1}$ counts in comparison with the data from the $24 \mathrm{~h}$ time point $\left({ }^{* *} P<0.05\right.$, $\left.{ }^{* * *} P<0.001\right)$.

a single species. However, in the setup described here, once a steady-state has been achieved (after around $24 \mathrm{~h}$ incubation) each inoculated species can be maintained at a constant titre, presumably reflecting the carrying capacity for each organism in the culture. Significantly, we show that even low-abundance species (represented by CA in our model) can be stably maintained, and that the presence of such species can have a major impact on the population trajectory of numerically more-abundant organisms such as SA, as well as inter-cellular signalling by PA.

The airways of people with CF have been shown to harbour a diverse polymicrobial community, comprising both bacteria and fungi (Sibley et al., 2006, 2008; Rogers et al., 2010a; Zhao et al., 2012; Carmody et al., 2013, 2015; Short et al., 2014; Boutin et al., 2015), and through the efforts of several teams, we now have a well-defined ASM for in vitro analyses. Indeed, PA grown in ASM has an almost identical gene expression profile compared with PA grown directly in sputum derived from CF patients (Turner et al., 2015). In spite of this, to date, there have been no reports describing the successful, long-term co-culture of CF-associated microbes in ASM. As we demonstrate in the current work, simply adding mixed-species inocula into ASM is not a recipe for the long-term maintenance of a stable population. Perhaps the best measure of the lack of progress on this front is seen when considering PA and SA. These two species are common in CF infections, and decades of work have revealed a wealth of knowledge about their physiology and nutritional requirements in axenic culture. However, until now, there have been no studies describing the successful long-term co-cultivation of these two species in vitro. One possible reason for this is that in iron limited conditions, PA lyses SA and uses the resulting lysate as a source of iron (Mashburn et al., 2005). By providing a continual supply of fresh media (which presumably mimics the unrelenting and exuberant production of airway secretions in the CF lung) we speculate that this nutritional limitation may be overcome.

The in vitro system described here offers a number of advantages. First, it is inexpensive to set up, making it accessible as a model to most researchers. Second, it is compliant with the "3Rs" (the replacement, refinement and reduction of animal research). Third, it is robust, as attested by the remarkably constant titres of each species following attainment of the steadystate condition. Fourth, early indications are that it can faithfully 


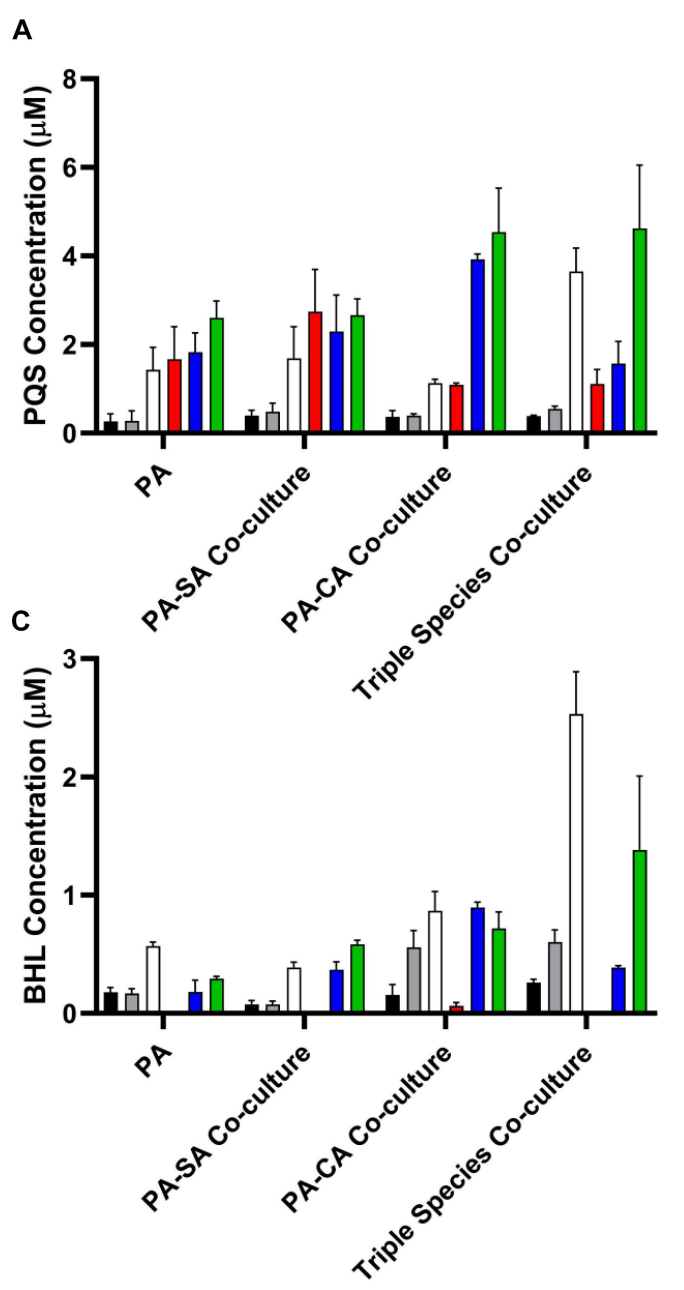

\section{B}

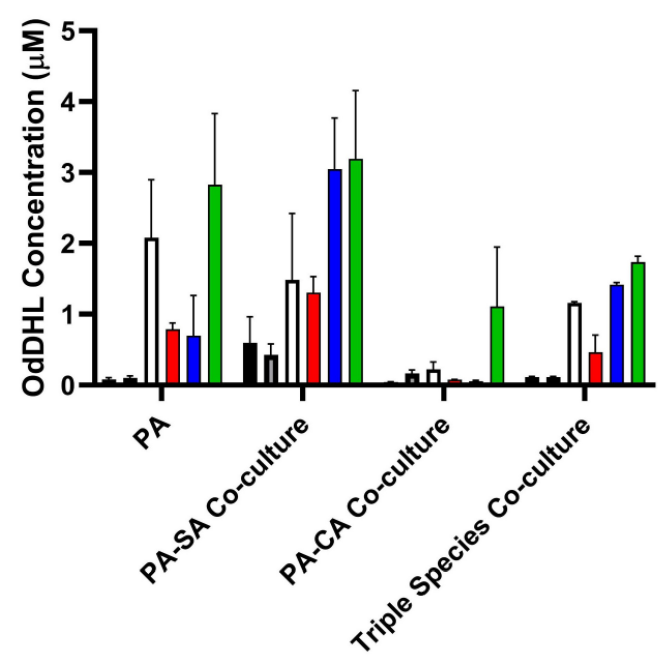

Continuous-flow (24

Hours)

Continuous-flow (96

Hours)

$\square$ Aerobic Batch (24 Hours)

$\square$ Aerobic Batch (96 Hours)

- Stirred Batch (24 Hours)

$\square$ Stirred Batch (96 Hours)

FIGURE 7 | Quorum sensing molecule accumulation in the different culture setups. Concentration of the indicated $P$. aeruginosa quorum sensing molecules in the supernatant of single-species or multi-species co-cultures after 24 and $96 \mathrm{~h}$ incubation (as indicated). (A) Pseudomonas quinolone signal (PQS);

(B) N-(3-oxododecanoyl)-L-homoserine lactone (OdDHL); (C) N-butanoyl-L-homoserine lactone (BHL). Data represented as mean \pm standard deviation of three independent experiments.

maintain species diversity when patient-derived CF sputum is being used to inoculate the system, and our progress on that aspect of the model will be published presently. Fifth, the system is far more defined and controlled than an animal model, allowing facile experimental perturbation. This experimental tractability means that we can address biological questions in a way that is just not possible with, e.g., animal models. For example, new species or defined mutants can be readily introduced to examine their impact on succession dynamics, and the action of antibiotics on the entire community can be accessed. We have also been exploring ways of modifying the setup to promote biofilm growth in the culture vessel, and again, these findings will be published in the near future.

Our in vitro setup is also subject to a number of perceived disadvantages. Unlike an animal model, it does not incorporate any immune response. This may be significant since the immune response would be expected to play a major role in clearance of microbes from the airways, and therefore exerts a selective pressure on the microbial community. Also, our model does not incorporate other types of host cell. This may be significant because in some circumstances (e.g., in patients carrying the DF508 CFTR mutation) the altered cell surface on the epithelia lining the airways has been implicated in promoting microbial colonisation (Campodónico et al., 2008). Mitigating these features, we note that few animal models accurately recapitulate the human CF airway environment, and aside from the difficulties associated with controlling and sampling such models, as far as we are aware, none of these models have yet been developed for maintaining a polymicrobial community of CF pathogens (O'Brien and Welch, 2019). One other potential disadvantage of our model is the requirement for continual flow. On the one hand, this is a feature that does allow maintenance of a stable steady-state community of microbes. On the other hand, even at low Q values, "washout" may prevent slow-growing species/variants from thriving, or key molecules from accumulating. For example, we noted that QS 


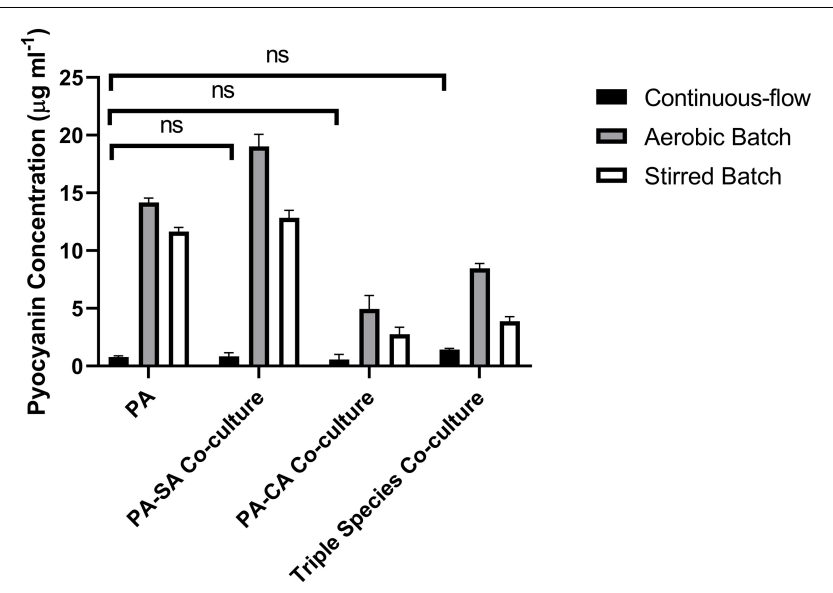

FIGURE 8 | Pyocyanin accumulation in the different culture setups. The concentration $\left(\mu \mathrm{g} \mathrm{mL}^{-1}\right.$ ) of pyocyanin in the supernatants of single-species and co-cultures after $96 \mathrm{~h}$ of incubation under: continuous-flow (black bars); aerobic batch (grey bars) and; stirred batch (white bars) culture conditions. Data represented as mean \pm standard deviation of three independent experiments, $P>0.05$ is considered no significant difference (ns).

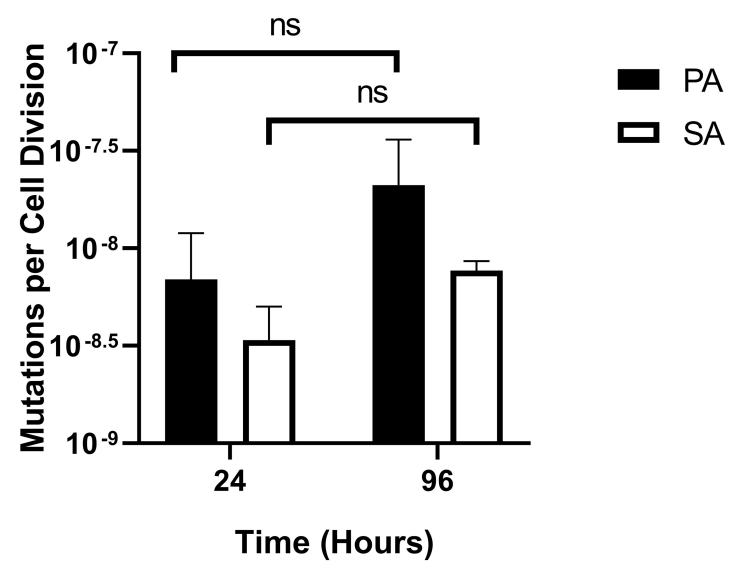

FIGURE 9 | Mutation rates of $P$. aeruginosa and $S$. aureus during co-culture in the continuous-flow setup. Assumed mutation rates of $P$. aeruginosa PAO1 (black bars) and S. aureus 25923 (white bars) in the continuous-flow culture vessel after 24 and $96 \mathrm{~h}$ of incubation. Mutation rates were calculated as the number of Rif ${ }^{R}$-conferring mutations per cell per cell division, calculated as described by Foster (2006). The bars represent the mean \pm standard deviation from three independent experiments. $P>0.05$ is considered not significantly different (ns).

molecules (and pyocyanin) fail to accumulate in the continuousflow system, whereas these compounds reached high levels in batch culture. The most likely explanation for this is simple washout (through continual dilution) of the QS signals. However, it should be noted that with $\mathrm{Q}=170 \mu \mathrm{L} \mathrm{min}-1$, it would take $>6 \mathrm{~h}$ to dilute the vessel contents by $50 \%$, and all the while, the contained culture continues to grow and elaborate more QS molecules. To put this into context, previous work has shown that QS molecules more than double their concentration in batch cultures in a $2 \mathrm{~h}$ period (Davenport et al., 2015), so assuming similar kinetics in ASM, these molecules should accumulate faster than they are diluted. If so, this suggests that QS plays a less important role in continuous-flow cultures than it does in batch cultures. The low steady-state concentrations of QS molecules documented here may also be advantageous (for the experimenter). First of all, the metabolic physiology of the community is defined and stable over the experiment's time course and we would not expect to see the bursts of metabolic activity which would normally accompany the accumulation of QS molecules in the post-quorate period (Davenport et al., 2015). Second, and if the effect(s) of QS molecules on community interactions does need to be examined, this can be easily be done through the addition of defined concentrations of exogenous QS molecules.

We conclude that the setup described here enables facile maintenance of PA, SA and CA (a Gram-negative bacterial species, a Gram-positive bacterial species and dimorphic fungus, respectively). Our approach provides a framework for potentially recapitulating the entire polymicrobial community associated with CF airway infections. The setup will provide leverage to access to key biological problems regarding inter-species interactions, the impact of antibiotics, and the impact that newlyintroduced species may have on the community trajectory.

\section{DATA AVAILABILITY STATEMENT}

The raw data supporting the conclusions of this manuscript will be made available by the authors, without undue reservation, to any qualified researcher.

\section{AUTHOR CONTRIBUTIONS}

TO'B and MW conceived and designed the work and revised the manuscript. TO'B executed the experiments, analysed the data, and drafted the manuscript.

\section{FUNDING}

This work was supported by a studentship (NC/P001564/1) from the NC3Rs to support TO'B, and consumables support from the UK Cystic Fibrosis Trust and British Lung Foundation.

\section{ACKNOWLEDGMENTS}

Andres Floto is acknowledged for helpful discussions and for providing the C. albicans.

\section{SUPPLEMENTARY MATERIAL}

The Supplementary Material for this article can be found online at: https://www.frontiersin.org/articles/10.3389/fmicb.2019. 02713/full\#supplementary-material 


\section{REFERENCES}

Antunes, L. C., Ferreira, R. B., Buckner, M. M., and Finlay, B. B. (2010). Quorum sensing in bacterial virulence. Microbiology 156(Pt 8), 2271-2282. doi: 10.1099/ mic.0.038794-0

Baldan, R., Cigana, C., Testa, F., Bianconi, I., De Simone, M., and Pellin, D. (2014). Adaptation of Pseudomonas aeruginosa in cystic fibrosis airways influences virulence of Staphylococcus aureus in vitro and murine models of co-infection. PLoS One 9:e89614. doi: 10.1371/journal.pone.00 89614

Barnabie, P. M., and Whiteley, M. (2015). Iron-mediated control of Pseudomonas aeruginosa-Staphylococcus aureus interactions in the cystic fibrosis lung. J. Bacteriol. 197, 2250-2251. doi: 10.1128/JB.00303-15

Biswas, L., Biswas, R., Schlag, M., Bertram, R., and Götz, F. (2009). Small-colony variant selection as a survival strategy for Staphylococcus aureus in the presence of Pseudomonas aeruginosa. Appl. Environ. Microbiol. 75, 6910-6912. doi: 10. 1128/AEM.01211-09

Bobadilla, J. L., Macek, M., Fine, J. P., and Farrell, P. M. (2002). Cystic fibrosis: a worldwide analysis of CFTR mutations-correlation with incidence data and application to screening. Hum. Mutat. 19, 575-606. doi: 10.1002/humu.10041

Bouchara, J. P., Symoens, F., Schwarz, C., and Chaturvedi, V. (2018). Fungal respiratory infections in cystic fibrosis (cf): recent progress and future research agenda. Mycopathologia 183, 1-5. doi: 10.1007/s11046-017-0241-6

Boucher, R. C. (2002). An overview of the pathogenesis of cystic fibrosis lung disease. Adv. Drug Delivery Rev. 54, 1359-1371. doi: 10.1016/S0169-409X(02) 00144-8

Boutin, S., Graeber, S. Y., Weitnauer, M., Panitz, J., Stahl, M., and Clausznitzer, D. (2015). Comparison of microbiomes from different niches of upper and lower airways in children and adolescents with cystic fibrosis. PLoS One 10:e0116029. doi: 10.1371/journal.pone.0116029

Bragonzi, A., Farulla, I., Paroni, M., Twomey, K. B., Pirone, L., and Lorè, N. I. (2012). Modelling co-infection of the cystic fibrosis lung by Pseudomonas aeruginosa and Burkholderia cenocepacia reveals influences on biofilm formation and host response. PLoS One 7:e52330. doi: 10.1371/journal.pone. 0052330

Campodónico, V. L., Gadjeva, M., Paradis-Bleau, C., Uluer, A., and Pier, G. B. (2008). Airway epithelial control of Pseudomonas aeruginosa infection in cystic fibrosis. Trends Mol. Med. 14, 120-133. doi: 10.1016/j.molmed.2008.01.002

Carmody, L. A., Zhao, J., Kalikin, L. M., LeBar, W., Simon, R. H., and Venkataraman, A. (2015). The daily dynamics of cystic fibrosis airway microbiota during clinical stability and at exacerbation. Microbiome 3:12. doi: 10.1186/s40168-015-0074-9

Carmody, L. A., Zhao, J., Schloss, P. D., Petrosino, J. F., Murray, S., and Young, V. B. (2013). Changes in cystic fibrosis airway microbiota at pulmonary exacerbation. Ann. Am. Thorac Soc. 10, 179-187. doi: 10.1513/AnnalsATS. 201211-107OC

Castric, P. A. (1975). Hydrogen cyanide, a secondary metabolite of Pseudomonas aeruginosa. Can. J. Microbiol. 21, 613-618. doi: 10.1139/m75-088

Conrad, D., Haynes, M., Salamon, P., Rainey, P. B., Youle, M., and Rohwer, F. (2013). Cystic fibrosis therapy: a community ecology perspective. Am. J. Respir. Cell Mol. Biol. 48, 150-156. doi: 10.1165/rcmb.2012-0059PS

Cugini, C., Morales, D. K., and Hogan, D. A. (2010). Candida albicans-produced farnesol stimulates Pseudomonas quinolone signal production in LasR-defective Pseudomonas aeruginosa strains. Microbiology 156(Pt 10), 3096-3107. doi: 10. 1099/mic.0.037911-0

Cystic Fibrosis Foundation (2019). Cystic Fibrosis Foundation (http://www.cff.org). Bethesda: Cystic Fibrosis Foundation

Cystic Fibrosis Mutation Database (2019). Cystic Fibrosis Mutation Database. Cystic Fibrosis Mutation Database: Bethesda.

Davenport, P. W., Griffin, J. L., and Welch, M. (2015). Quorum sensing is accompanied by global metabolic changes in the opportunistic human pathogen Pseudomonas aeruginosa. J. Bacteriol. 197, 2072-2082. doi: 10.1128/JB.02 557-14

Dekimpe, V., and Déziel, E. (2009). Revisiting the quorum-sensing hierarchy in Pseudomonas aeruginosa: the transcriptional regulator RhlR regulates LasR-specific factors. Microbiology 155(Pt 3), 712-723. doi: 10.1099/mic.0.02 2764-0
Dettman, J. R., Sztepanacz, J. L., and Kassen, R. (2016). The properties of spontaneous mutations in the opportunistic pathogen Pseudomonas aeruginosa. BMC Genomics 17:27. doi: 10.1186/s12864-015-2244-3

Duan, K., Dammel, C., Stein, J., Rabin, H., and Surette, M. G. (2003). Modulation of Pseudomonas aeruginosa gene expression by host microflora through interspecies communication. Mol. Microbiol. 50, 1477-1491. doi: 10.1046/j. 1365-2958.2003.03803.x

Elborn, J. S. (2016). Cystic fibrosis. Lancet 388, 2519-2531. doi: 10.1016/S01406736(16)00576-6

Elias, S., and Banin, E. (2012). Multi-species biofilms: living with friendly neighbors. FEMS Microbiol. Rev. 36, 990-1004. doi: 10.1111/j.1574-6976.2012. 00325.x

Filkins, L. M., Graber, J. A., Olson, D. G., Dolben, E. L., Lynd, L. R., and Bhuju, S. (2015). Coculture of Staphylococcus aureus with Pseudomonas aeruginosa Drives S. aureus towards fermentative metabolism and reduced viability in a cystic fibrosis model. J. Bacteriol. 197, 2252-2264. doi: 10.1128/JB.00059-15

Fletcher, M. P., Diggle, S. P., Cámara, M., and Williams, P. (2007). Biosensor-based assays for PQS, HHQ and related 2-alkyl-4-quinolone quorum sensing signal molecules. Nat. Protoc. 2, 1254-1262. doi: 10.1038/nprot.2007.158

Foster, P. L. (2006). Methods for determining spontaneous mutation rates. Methods Enzymol. 409, 195-213. doi: 10.1016/S0076-6879(05)09012-9

Fugère, A., Lalonde Séguin, D., Mitchell, G., Déziel, E., Dekimpe, V., and Cantin, A. M. (2014). Interspecific small molecule interactions between clinical isolates of Pseudomonas aeruginosa and Staphylococcus aureus from adult cystic fibrosis patients. PLoS One 9:e86705. doi: 10.1371/journal.pone.0086705

Gambello, M. J., Kaye, S., and Iglewski, B. H. (1993). LasR of Pseudomonas aeruginosa is a transcriptional activator of the alkaline protease gene (apr) and an enhancer of exotoxin A expression. Infect Immun. 61, 1180-1184.

Ghio, A. J., Roggli, V. L., Soukup, J. M., Richards, J. H., Randell, S. H., and Muhlebach, M. S. (2013). Iron accumulates in the lavage and explanted lungs of cystic fibrosis patients. J. Cyst. Fibros 12, 390-398. doi: 10.1016/j.jcf.2012.10.010

Gillum, A. M., Tsay, E. Y., and Kirsch, D. R. (1984). Isolation of the Candida albicans gene for orotidine- $5^{\prime}$-phosphate decarboxylase by complementation of S. cerevisiae ura3 and E. coli pyrF mutations. Mol. Gen. Genet. 198, 179-182. doi: $10.1007 /$ bf00328721

Goss, C. H., and Muhlebach, M. S. (2011). Review: Staphylococcus aureus and MRSA in cystic fibrosis. J. Cyst. Fibros 10, 298-306. doi: 10.1016/j.jcf.2011.06. 002

Grasemann, H., Ioannidis, I., Tomkiewicz, R. P., de Groot, H., Rubin, B. K., and Ratjen, F. (1998). Nitric oxide metabolites in cystic fibrosis lung disease. Arch. Dis. Child 78, 49-53. doi: 10.1136/adc.78.1.49

Hibbing, M. E., Fuqua, C., Parsek, M. R., and Peterson, S. B. (2010). Bacterial competition: surviving and thriving in the microbial jungle. Nat. Rev. Microbiol. 8, 15-25. doi: 10.1038/nrmicro2259

Hoffman, L. R., Déziel, E., D’Argenio, D. A., Lépine, F., Emerson, J., and McNamara, S. (2006). Selection for Staphylococcus aureus small-colony variants due to growth in the presence of Pseudomonas aeruginosa. Proc. Natl. Acad. Sci. U.S.A. 103, 19890-19895. doi: 10.1073/pnas.0606756104

Hogan, D. A., Vik, A., and Kolter, R. (2004). A Pseudomonas aeruginosa quorumsensing molecule influences Candida albicans morphology. Mol. Microbiol. 54, 1212-1223. doi: 10.1111/j.1365-2958.2004.04349.x

Holcombe, L. J., McAlester, G., Munro, C. A., Enjalbert, B., Brown, A., and Gow, J. P. (2010). Pseudomonas aeruginosa secreted factors impair biofilm development in Candida albicans. Microbiology 156(Pt 5), 1476-1486. doi: 10. 1099/mic.0.037549-0

Holloway, B. W. (1955). Genetic recombination in Pseudomonas aeruginosa. J. Gen. Microbiol. 13, 572-581. doi: 10.1099/00221287-13-3-572

Ibberson, C. B., Stacy, A., Fleming, D., Dees, J. L., Rumbaugh, K., and Gilmore, M. S. (2017). Co-infecting microorganisms dramatically alter pathogen gene essentiality during polymicrobial infection. Nat. Microbiol. 2:17079. doi: 10 . 1038/nmicrobiol.2017.79

Jones, K. L., Hegab, A. H., Hillman, B. C., Simpson, K. L., Jinkins, P. A., and Grisham, M. B. (2000). Robbins: elevation of nitrotyrosine and nitrate concentrations in cystic fibrosis sputum. Pediatr. Pulmonol. 30, 79-85. doi: 10.1002/1099-0496(200008)30:2<79::aid-ppul1>3.0.co;2-1

Jorth, P., Ehsan, Z., Rezayat, A., Caldwell, E., Pope, C., and Brewington, J. J. (2019). Direct lung sampling indicates that established pathogens dominate 
early infections in children with cystic fibrosis. Cell Rep. 27:1190-1204.e3. doi: 10.1016/j.celrep.2019.03.086

Kirchner, S., Fothergill, J. L., Wright, E. A., James, C. E., Mowat, E., and Winstanley, C. (2012). Use of artificial sputum medium to test antibiotic efficacy against Pseudomonas aeruginosa in conditions more relevant to the cystic fibrosis lung. J. Vis. Exp. 5:e3857. doi: 10.3791/3857

Knight, M., Hartman, P. E., Hartman, Z., and Young, V. M. (1979). A new method of preparation of pyocyanin and demonstration of an unusual bacterial sensitivity. Anal. Biochem. 95, 19-23. doi: 10.1016/0003-2697(79)90179-9

Korgaonkar, A., Trivedi, U., Rumbaugh, K. P., and Whiteley, M. (2013). Community surveillance enhances Pseudomonas aeruginosa virulence during polymicrobial infection. Proc. Natl. Acad. Sci. U.S.A. 110, 1059-1064. doi: 10. 1073/pnas. 1214550110

Lau, G. W., Hassett, D. J., Ran, H., and Kong, F. (2004). The role of pyocyanin in Pseudomonas aeruginosa infection. Trends Mol. Med. 10, 599-606. doi: 10.1016/ j.molmed.2004.10.002

Leekha, S., Terrell, C. L., and Edson, R. S. (2011). General principles of antimicrobial therapy. Mayo Clin. Proc. 86, 156-167. doi: 10.4065/mcp.2010. 0639

Limoli, D. H., Yang, J., Khansaheb, M. K., Helfman, B., Peng, L., and Stecenko, A. A. (2016). Staphylococcus aureus and Pseudomonas aeruginosa co-infection is associated with cystic fibrosis-related diabetes and poor clinical outcomes. Eur. J. Clin. Microbiol. Infect Dis. 35, 947-953. doi: 10.1007/s10096-016-2621-0

Lopes, S. P., Azevedo, N. F., and Pereira, M. O. (2017). Developing a model for cystic fibrosis sociomicrobiology based on antibiotic and environmental stress. Int. J. Med. Microbiol. 307, 460-470. doi: 10.1016/j.ijmm.2017.09.018

Lopes, S. P., Ceri, H., Azevedo, N. F., and Pereira, M. O. (2012). Antibiotic resistance of mixed biofilms in cystic fibrosis: impact of emerging microorganisms on treatment of infection. Int. J. Antimicrob. Agents 40, 260-263. doi: 10.1016/j.ijantimicag.2012.04.020

Lyczak, J. B., Cannon, C. L., and Pier, G. B. (2002). Lung infections associated with cystic fibrosis. Clin. Microbiol. Rev. 15, 194-222. doi: 10.1128/cmr.15.2.194-222. 2002

Machan, Z. A., Taylor, G. W., Pitt, T. L., Cole, P. J., and Wilson, R. (1992). 2Heptyl-4-hydroxyquinoline $\mathrm{N}$-oxide, an antistaphylococcal agent produced by Pseudomonas aeruginosa. J. Antimicrob. Chemother. 30, 615-623. doi: 10.1093/ jac/30.5.615

Magalhães, A. P., Lopes, S. P., and Pereira, M. O. (2016). Insights into cystic fibrosis polymicrobial consortia: the role of species interactions in biofilm development, phenotype, and response to in-use antibiotics. Front. Microbiol. 7:2146. doi: $10.3389 /$ fmicb.2016.02146

Makovcova, J., Babak, V., Kulich, P., Masek, J., Slany, M., and Cincarova, L. (2017). Dynamics of mono- and dual-species biofilm formation and interactions between Staphylococcus aureus and Gram-negative bacteria. Microb. Biotechnol. 10, 819-832. doi: 10.1111/1751-7915.12705

Mashburn, L. M., Jett, A. M., Akins, D. R., and Whiteley, M. (2005). Staphylococcus aureus serves as an iron source for Pseudomonas aeruginosa during in vivo coculture. J. Bacteriol. 187, 554-566. doi: 10.1128/JB.187.2.554-566.2005

McAlester, G., O'Gara, F., and Morrissey, J. P. (2008). Signal-mediated interactions between Pseudomonas aeruginosa and Candida albicans. J. Med. Microbiol. 57(Pt 5), 563-569. doi: 10.1099/jmm.0.47705-0

Morales, D. K., Jacobs, N. J., Rajamani, S., Krishnamurthy, M., Cubillos-Ruiz, J. R., and Hogan, D. A. (2010). Antifungal mechanisms by which a novel Pseudomonas aeruginosa phenazine toxin kills Candida albicans in biofilms. Mol. Microbiol. 78, 1379-1392. doi: 10.1111/j.1365-2958.2010.07414.x

Nguyen, A. T., Jones, J. W., Ruge, M. A., Kane, M. A., and Oglesby-Sherrouse, A. G. (2015). Iron depletion enhances production of antimicrobials by Pseudomonas aeruginosa. J. Bacteriol. 197, 2265-2275. doi: 10.1128/JB.00072-15

Nguyen, A. T., and Oglesby-Sherrouse, A. G. (2016). Interactions between Pseudomonas aeruginosa and Staphylococcus aureus during co-cultivations and polymicrobial infections. Appl. Microbiol. Biotechnol. 100, 6141-6148. doi: 10. 1007/s00253-016-7596-3

Noto, M. J., Burns, W. J., Beavers, W. N., and Skaar, E. P. (2017). Mechanisms of pyocyanin toxicity and genetic determinants of resistance in Staphylococcus aureus. J. Bacteriol. 199:e221-17. doi: 10.1128/JB.00221-17

O’Brien, , T. J., and Welch, M. (2019). Recapitulation of polymicrobial communities associated with cystic fibrosis airway infections: a perspective. Future Microbiol. (in press). doi: 10.2217/fmb-2019-0200
Palmer, K. L., Aye, L. M., and Whiteley, M. (2007). Nutritional cues control Pseudomonas aeruginosa multicellular behavior in cystic fibrosis sputum. J. Bacteriol. 189, 8079-8087. doi: 10.1128/JB.01138-07

Park, J. H., Lee, J. H., Cho, M. H., Herzberg, M., and Lee, J. (2012). Acceleration of protease effect on Staphylococcus aureus biofilm dispersal. FEMS Microbiol. Lett. 335, 31-38. doi: 10.1111/j.1574-6968.2012.02635.x

Peters, B. M., Jabra-Rizk, M. A. O., May, G. A., Costerton, J. W., and Shirtliff, M. E. (2012). Polymicrobial interactions: impact on pathogenesis and human disease. Clin. Microbiol. Rev. 25, 193-213. doi: 10.1128/CMR.00013-11

Quinn, R. A., Lim, Y. W., Maughan, H., Conrad, D., Rohwer, F., and Whiteson, K. L. (2014). Biogeochemical forces shape the composition and physiology of polymicrobial communities in the cystic fibrosis lung. MBio 5:e956-13. doi: $10.1128 / \mathrm{mBio} .00956-13$

Rajan, S., and Saiman, L. (2002). Pulmonary infections in patients with cystic fibrosis. Semin Respir Infect 17, 47-56. doi: 10.1053/srin.2002.31690

Rogers, G. B., Carroll, M. P., and Bruce, K. D. (2009). Studying bacterial infections through culture-independent approaches. J. Med. Microbiol. 58(Pt 11), 14011418. doi: 10.1099/jmm.0.013334-0

Rogers, G. B., Hoffman, L. R., Whiteley, M., Daniels, T. W., Carroll, M. P., and Bruce, K. D. (2010a). Revealing the dynamics of polymicrobial infections: implications for antibiotic therapy. Trends Microbiol. 18, 357-364. doi: 10.1016/ j.tim.2010.04.005

Rogers, G. B., Stressmann, F. A., Walker, A. W., Carroll, M. P., and Bruce, K. D. (2010b). Lung infections in cystic fibrosis: deriving clinical insight from microbial complexity. Expert Rev. Mol. Diagn 10, 187-196. doi: 10.1586/erm. 09.81

Rüger, M., Ackermann, M., and Reichl, U. (2014). Species-specific viability analysis of Pseudomonas aeruginosa, Burkholderia cepacia and Staphylococcus aureus in mixed culture by flow cytometry. BMC Microbiol. 14:56. doi: 10.1186/14712180-14-56

Schaaff, F., Reipert, A., and Bierbaum, G. (2002). An elevated mutation frequency favors development of vancomycin resistance in Staphylococcus aureus. Antimicrob. Agents Chemother. 46, 3540-3548. doi: 10.1128/aac.46.11.35403548.2002

Schuster, M., and Greenberg, E. P. (2006). A network of networks: quorum-sensing gene regulation in Pseudomonas aeruginosa. Int. J. Med. Microbiol. 296, 73-81. doi: 10.1016/j.ijmm.2006.01.036

Short, F. L., Murdoch, S. L., and Ryan, R. P. (2014). Polybacterial human disease: the ills of social networking. Trends Microbiol. 22, 508-516. doi: 10.1016/j.tim. 2014.05.007

Sibley, C. D., Parkins, M. D., Rabin, H. R., Duan, K., Norgaard, J. C., and Surette, M. G. (2008). A polymicrobial perspective of pulmonary infections exposes an enigmatic pathogen in cystic fibrosis patients. Proc. Natl. Acad. Sci. U.S.A. 105, 15070-15075. doi: 10.1073/pnas.0804326105

Sibley, C. D., Rabin, H., and Surette, M. G. (2006). Cystic fibrosis: a polymicrobial infectious disease. Future Microbiol. 1, 53-61. doi: 10.2217/17460913.1.1.53

Smith, R. S., and Iglewski, B. H. (2003). P. aeruginosa quorum-sensing systems and virulence. Curr. Opin. Microbiol. 6, 56-60. doi: 10.1016/s1369-5274(03)00 008-0

Spasenovski, T., Carroll, M. P., Lilley, A. K., Payne, M. S., and Bruce, K. D. (2010). Modelling the bacterial communities associated with cystic fibrosis lung infections. Eur. J. Clin. Microbiol. Infect Dis. 29, 319-328. doi: 10.1007/s10096009-0861-y

Tate, S., MacGregor, G., Davis, M., Innes, J. A., and Greening, A. P. (2002). Airways in cystic fibrosis are acidified: detection by exhaled breath condensate. Thorax 57, 926-929. doi: 10.1136/thorax.57.11.926

Thomas, P., Sekhar, A. C., Upreti, R., Mujawar, M. M., and Pasha, S. S. (2015). Optimization of single plate-serial dilution spotting (SP-SDS) with sample anchoring as an assured method for bacterial and yeast cfu enumeration and single colony isolation from diverse samples. Biotechnol. Rep. 8, 45-55. doi: 10.1016/j.btre.2015.08.003

Treangen, T. J., Maybank, R. A., Enke, S., Friss, M. B., Diviak, L. F., and Karaolis, D. K. (2014). Complete genome sequence of the quality control strain Staphylococcus aureus subsp. aureus ATCC 25923. Genome Announc. 2:e1110-14. doi: 10.1128/genomeA.01110-14

Turner, K. H., Wessel, A. K., Palmer, G. C., Murray, J. L., and Whiteley, M. (2015). Essential genome of Pseudomonas aeruginosa in cystic fibrosis sputum. Proc. Natl. Acad. Sci. U.S.A. 112, 4110-4115. doi: 10.1073/pnas. 1419677112 
Voggu, L., Schlag, S., Biswas, R., Rosenstein, R., Rausch, C., and Götz, F. (2006). Microevolution of cytochrome bd oxidase in Staphylococci and its implication in resistance to respiratory toxins released by Pseudomonas. J. Bacteriol. 188, 8079-8086. doi: 10.1128/JB.00858-06

Williams, C., Ranjendran, R., and Ramage, G. (2016). Pathogenesis of fungal infections in cystic fibrosis. Curr. Fungal Infect Rep. 10, 163-169. doi: 10.1007/ s12281-016-0268-Z

Winson, M. K., Swift, S., Fish, L., Throup, J. P., Jørgensen, F., and Chhabra, S. R. (1998). Construction and analysis of luxCDABE-based plasmid sensors for investigating $\mathrm{N}$-acyl homoserine lactone-mediated quorum sensing. FEMS Microbiol. Lett. 163, 185-192. doi: 10.1111/j.1574-6968.1998.tb13044.x

Worlitzsch, D., Tarran, R., Ulrich, M., Schwab, U., Cekici, A., and Meyer, K. C. (2002). Effects of reduced mucus oxygen concentration in airway Pseudomonas infections of cystic fibrosis patients. J. Clin. Invest 109, 317-325. doi: 10.1172/ JCI13870

Zago, C. E., Silva, S., Sanitá, P. V., Barbugli, P. A., Dias, C. M., and Lordello, V. B. (2015). Dynamics of biofilm formation and the interaction between Candida albicans and methicillin-susceptible (MSSA) and -resistant Staphylococcus aureus (MRSA). PLoS One 10:e123206. doi: 10.1371/journal.pone.0123206

Zhao, J., Schloss, P. D., Kalikin, L. M., Carmody, L. A., Foster, B. K., and Petrosino, J. F. (2012). Decade-long bacterial community dynamics in cystic fibrosis airways. Proc. Natl. Acad. Sci. U.S.A. 109, 5809-5814. doi: 10.1073/pnas. 1120577109

Conflict of Interest: The authors declare that the research was conducted in the absence of any commercial or financial relationships that could be construed as a potential conflict of interest.

Copyright (c) 2019 O'Brien and Welch. This is an open-access article distributed under the terms of the Creative Commons Attribution License (CC BY). The use, distribution or reproduction in other forums is permitted, provided the original author(s) and the copyright owner(s) are credited and that the original publication in this journal is cited, in accordance with accepted academic practice. No use, distribution or reproduction is permitted which does not comply with these terms. 